Bulgarian Academy of Sciences. Space Research and Technology Institute.

Aerospace Research in Bulgaria. 33, 2021, Sofia

DOI: https://doi.org/10.3897/arb.v33.e08

\title{
INTERFEROMETRIC PROCESSING OF TANDEM-X BISTATIC SAR DATA USING GAMMA - IMPLEMENTATION OUTLINE
}

\section{Zlatomir Dimitrov}

\author{
Space Research and Technology Institute - Bulgarian Academy of Sciences \\ e-mail: zlatomir.dimitrov@space.bas.bg
}

Keywords: GAMMA Software, SAR, Interferometry, Interferometry Processing

\begin{abstract}
This article represents a showcase of two coding approaches with GAMMA, used to calculate topographic and differential phases from high resolution TanDEM-X bistatic data, provided by DLR. The first implementation approach comprises "BASH" scripting in Linux environment, having direct control of the GAMMA executables. The second approach is the utilisation of the PyroSAR framework, via GAMMA-API, in Python environment. Two spatial resolution scales are used - of 4 and 12 metres, to test the feasibility of TanDEM-X InSAR output products in mountainous forests in the rugged region. The first approach allowed thorough processing with abundant GAMMA output, whereas the high scale PyroSAR framework via GAMMA-API showed a fast implementation. Comparison over 4 and $12 \mathrm{~m}$ spatial resolution products showed good feasibility with strong influence from topography. Intense multi-looking resolved better connection of coherence amplitude to the volume decorrelation in canopy, despite preserving high resolution reveals plenty of details in land cover. The differential height calculation, without phase unwrapping, showed its significance in data processing over mountainous regions. Intensities normalisation and terrain flattening showed good performance in both resolution scales. Finally, utilisation of GAMMA in InSAR processing of high resolution TanDEM-X bistatic SAR data showed good feasibility and flexibility to derive interferometric products.
\end{abstract}

\section{Introduction}

Applying SAR Interferometry is promising in survey natural environment and has been widely assessed in scientific studies, pointing out Earth crust deformation after hazards, derivation of DEMs in various areas, studying forest canopy in respect to different environmental conditions [1,2] Recent years, considering forestry domain, plausible results in respect to the microwave band are provided from the Japanese L-band system ALOS PALSAR for assessing forest biomass and canopy height via POLinSAR modeling [3, 4]; via InSAR coherence on large scale boreal forest [5]; providing time series acquisitions to estimate large scale mapping of growing stocks either via L-band [6] or via C-band by ESA ENVISAT [7]; and the DLR's X-band bi-satellite system TanDEM-X, which 
overcomes the limitation of the temporal decorrelation, where the bistatic coherence is directly linked with the forest biomass $[8,9]$. Also, this allows forest modelling using POLinSAR-scattering models, via model inversion with two baselines [10]. Considering SAR remote sensing software, various graphical based products, both freeware and commercial, have been developed in recent years -

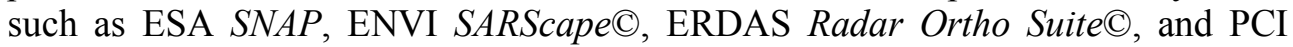
Geomatics $S A R$ polarimetry workstation $\subset$. In those software products, various InSAR processing workflows are implemented, comprising - direct calculation of DEM, Coherence change detection, or particular DInSAR workflows, providing more or less ease of use. Aiming at the SAR data and systems, the desired results from interferometric processing via provided tool-boxes, are not always as accurate as are needed. An example from the practice is the impossibility to adjust the particular intermediate parameter during processing, to react more adequate to the physical and environmental conditions of the observed area. In the sake of that, in particular to remote sensing studies useful approach could be provided by software GAMMAC, developed by Swiss Company "GAMMA Remote Sensing and Research $A G$ ", and available in Friedrich-Schiller-Universität-JENA, Institut für Geographie, Lehrstuhl für Fernerkundung. Coding with GAMMA offers professional attitude to the SAR processing tasks, where executable binaries from supported toolbox could be implemented in Linux-shell environment (BASH), or embedded in Python code.

This study demonstrates the use of GAMMA software for InSAR processing according to the use of two different method approaches. This constitutes of practical utilization of GAMMA using InSAR technology on provided SAR data from TanDEM-X, implemented in shell BASH scripts and open-source Python framework PyroSAR. Motivation is to utilise both software implementation and to test for the first time before the Bulgarian scientific community the interferometric processing of high resolution TanDEM-X bistatic data.

The general task is to derive the topographic and differential phases from bistatic X-band SAR data from TanDEM-X over mountainous forest in Bulgaria, along the presence of topography and different types of land cover. Therefore, following tasks are formulated in this study:

- Elaboration of optimal implementation chains using GAMMA software package, for interferometric processing of TanDEM-X data,

- Clarification of the InSAR workflow via specific implementation chains,

- Utilize PyroSAR framework, especially the GAMMA-API, in TanDEM-X bistatic data processing

- $\quad$ Calculate terrain flattened intensities by elimination of topography [11];

- Calculate bistatic Coherence from TanDEM-X data;

- Calculate Topographic phase, with high spatial resolution; 
- Calculate Differential phase, related to relevant phase center height from the referent DEM.

\section{Data and study area}

According to formulated aims and tasks the high resolution data from German satellite system - TanDEM-X provided by German Space Agency (DLR) was used in this study. Milestone is that provided SAR data are bistatic simultaneous acquisition, thus resulting elimination of the temporal decorrelation from InSAR pair. In the contribution to the scope of the study, high resolution with unprecedented accuracy Digital Elevation Model (DEM) from TanDEM-X “TanDEM-X DEM12” was used, provided by DLR.

\section{SAR data}

\section{TanDEM-X bi-satellite system}

The primary objective of TanDEM-X mission is to map terrain topography by generating a digital elevation model (DEM) on a global scale, with unprecedented accuracy employing bistatic acquisitions [12]. This is achieved by a couple of twin satellites - TerraSAR-X and TanDEM-X flying in a Sunsynchronous orbit in close formation, with varying baseline separation in between. A key feature of TanDEM-X is the bistatic acquisition that employs to eliminate the temporal decorrelation factor from interferometric coherence. Operational modes of acquisition that are currently operated by TanDEM-X bistatic system, are - bistatic, monostatic and alternating bistatic, which comes in combination with imaging modes of - ScanSAR, Stripmap and Spotlight with respect to resolution.

\section{Used TanDEM-X bistatic SAR data}

A single-pol TanDEM-X SAR dataset over the test site - TS21, provided by DLR in terms of CoSSC data proposal, was used to test outlined methodology. The operating mode is Alternating Bistatic, which constitutes two bistatic acquisitions - 'ALT1' and 'ALT2', and one pursuit monostatic acquisition 'MONO', packed in three separate dims-catalogs; it is spring acquisition, with specifications as follows:

Table 1. Specifications of the TanDEM-X SAR data acquisition

\begin{tabular}{|l|c|l|c|}
\hline AOI & TS21 & Average Coherence & 0.7506 \\
\hline Product & TDM1_COS_SM & Along-track Baseline (I) [m] & -184.9802 \\
\hline Orbit & DESCENDING & Effective Baseline (ـ) [m] & 150.9871 \\
\hline Date \& time & 2018-05-02, T04:36 & Ambiguity height [m] & 48.3917 \\
\hline Acquisition & Alternating Bistatic & Incidence Angle [deg] & 39.2733 \\
\hline Polarisation & HHHH & Range pixel spacing [m] & 1.3641 \\
\hline $\begin{array}{l}\text { Band, freq. } \\
\text { [GHz] }\end{array}$ & "X”, 5.589 & Azimuth pixel spacing [m] & 2.4277 \\
\hline
\end{tabular}


Each dataset is compiled in tar archive as a dims-catalog, with structure as follows:

- The first level - compulsory hierarchical tree with common supporting data of the bistatic acquisition, with the two acquisitions from TerraSAR$\mathrm{X} /$ TanDEM-X satellites, where in bistatic configuration first is transmitting (BTX1) and the second one is receiving only (BRX2).

- Second level - tree structure of the particular satellite's measurement, where:

- Annotation xml-files with various measurement metadata,

- Various auxiliary raster of previews for GoogleEarth,

- Measurements as a COS-file, with respect to polarisation,

- Various preview raster and other supporting information.

Table 2. Structure levels of the DIMS-catalog, with compiled TanDEM-X data

\begin{tabular}{|c|c|c|}
\hline \multicolumn{3}{|c|}{ The first level structure } \\
\hline \multirow{6}{*}{$\begin{array}{l}\text { (TDM.SAR.COSSC) } \\
\text { (spc.number) }\end{array}$} & \multirow{6}{*}{$\begin{array}{l}\text { TDM1_SAR_COS_ALT1_S } \\
\text { M_S_SRA_20180502T043648 } \\
\text { 20180502T043651 }\end{array}$} & COMMON_ANNOTATION \\
\hline & & COMMON_AUXRASTER \\
\hline & & COMMON_IMAGEDATA \\
\hline & & COMMON_PREVIEW \\
\hline & & $\begin{array}{l}\text { TDX1_SAR_SSC_BRX2_S } \\
\text { M_S_SRA_20180502T043648 } \\
\text { 20180502T043651 }\end{array}$ \\
\hline & & $\begin{array}{l}\text { TSX1_SAR_SSC_BTX1_SM } \\
\text { S_SRA_20180502T043648_- } \\
\text { 20180502T043651 }\end{array}$ \\
\hline \multicolumn{3}{|c|}{ Second level structure: } \\
\hline \multirow{5}{*}{$\begin{array}{l}\text { TDX1_SAR_SSC_BRX2_S } \\
\text { M_S_SRA_20180502T043648 } \\
\text { 20180502T043651 }\end{array}$} & ANNOTATION & ${ }^{*} . \mathrm{xml}$ \\
\hline & AUXRASTER & $*$.tif, *tif.kml \\
\hline & IMAGEDATA & $\begin{array}{l}\text { IMAGE_HH_SRA_stripFar_0 } \\
\text { 13.cos }\end{array}$ \\
\hline & PREVIEW & $*$.tif \\
\hline & SUPPORT & *.xsd \\
\hline
\end{tabular}

Both acquisitions from TerraSAR-X/TanDEM-X during pre-processing in DLR are precisely co-registered in slant-range geometry, thus formulating the CoSSC data format. Each acquisition is structured in a binary file in COSAR file format, formulated as 32-bit floating point Big-Endian data, organised in row order starting from the first to the last one. Relative to orbit the acquired image are normally flipped on North-South or East-West direction. A raw overview of the interferometric pair is also provided from the TanDEM-X pre-processor at DLR, including - intensity, coherence and interferometric phase along the scene, as shown on Fig. 1. 
(A) Intensity

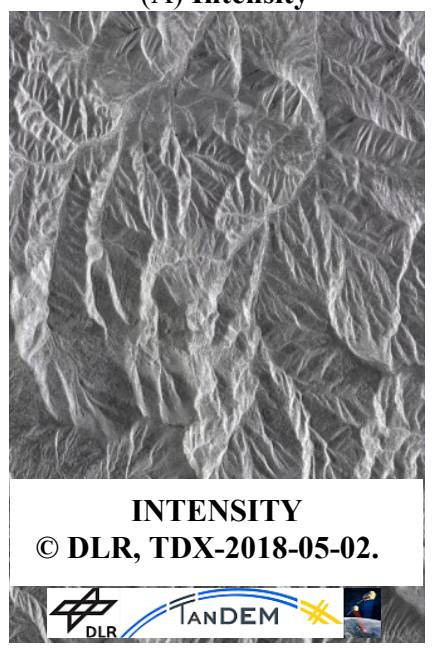

(B) Coherence

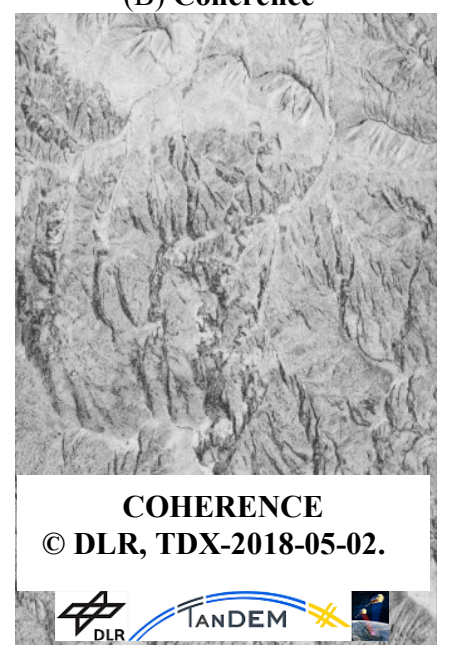

(C) Interferometric phase

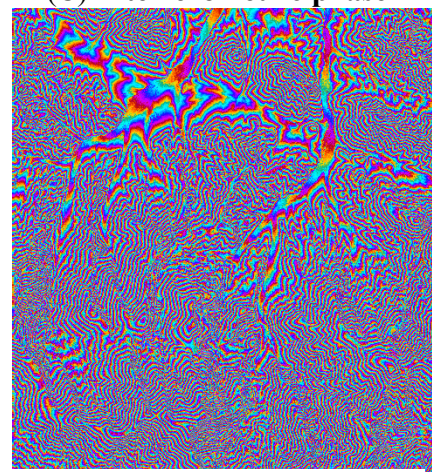

INT. PHASE

(C) DLR, TDX-2018-05-02.

Fig. 1. Provided quick-look images of the interferometric pair of the bistatic acquisition, from TanDEM-X; CDLR

\section{DEM data}

\section{High resolution "TanDEM-X World DEM12" reference DEM product}

The TanDEM-X World DEM12@ was elaborated according to conducted from DLR World DEM campaign, from 2010 to 2014, using a particular set of parameters with respect to topography and land cover $[12,13]$. It is acquired in the bistatic InSAR stripmap operational acquisition mode in single - HH polarisation. During whole four global acquisitions trade-off in values of ambiguity height and therefore effective baseline is made, map with sufficient accuracy for whole types of topography on earth. Most acquisitions with varying ambiguity heights and combination of ascending / descending nodes are over mountainous areas and tropical forests, held in the last global acquisitions. The final DEM product achieves absolute vertical accuracy of less than $10[\mathrm{~m}]$ for $90 \%$ of the Earth's surface, and relative vertical accuracy less than $2[\mathrm{~m}]$ for $10 \%$ of the Earth's surface (HRTI-3 DEM definition) [14].

\section{Used "TanDEM-X DEM12" data}

The innovative high accuracy TanDEM-X DEM12 product was used as a reference DEM, in the scope of a DLR's DEM-proposal, provided from the German Space Agency (DLR) in support to the study with the delivered TanDEM$\mathrm{X}$ bistatic data. An example is shown below of the DEM tile over North-West Bulgaria over mountain massif of "Stara Planina". Each tile constitutes at least - 
35 TanDEM-X acquisitions, with ascending/descending orbits, with a spatial resolution of 0.4 [arcsec] - or c.a. $12 \mathrm{~m}$ in UTM projection, shown in Fig. 2.
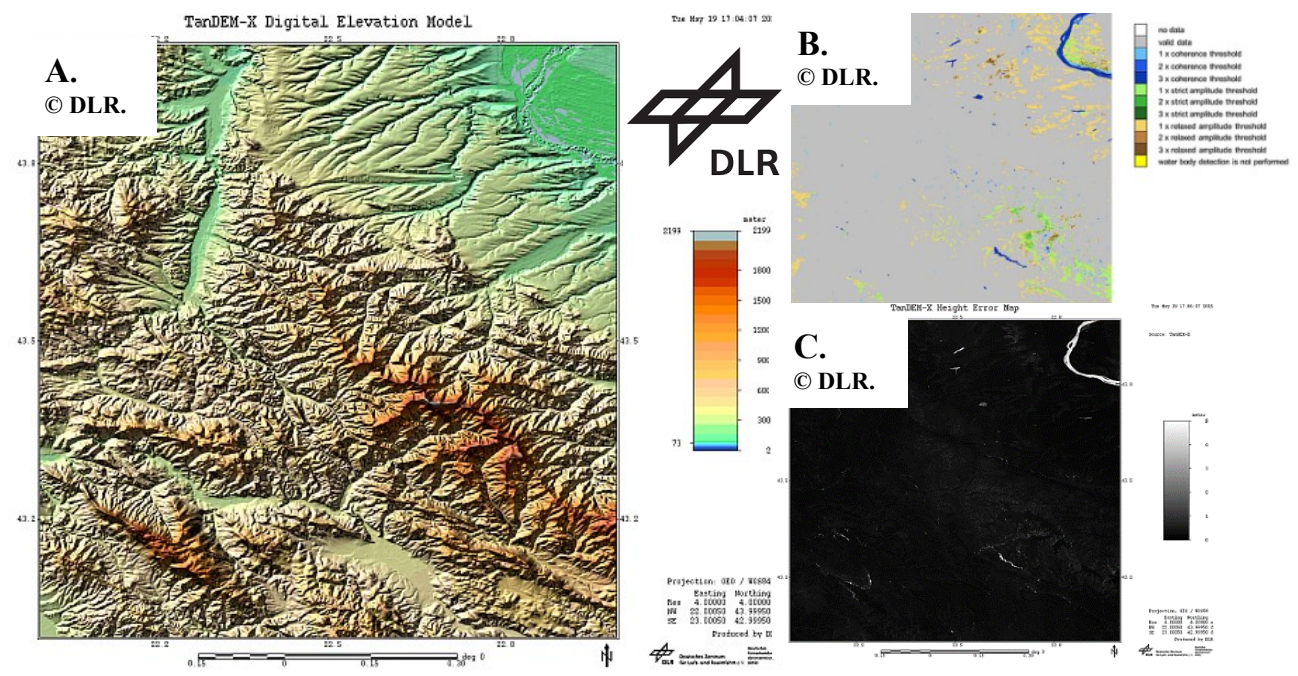

Fig. 2. Tile of the TanDEM-X DEM12 Product (A), height error map (B) and coherence thresholds, showing inconsistencies $(C)$ - preview of provided DEM tile, located in NW Bulgaria over Test sites -

20/21/22, in SFE "Chuprene", SFE "Mijur" and SFE "Govezhda” respectively. Source - CDLR

\section{Study area}

The selected test site is part from the PhD study of the author, with index TS21 "Chuprene". The test site is located in the Bulgarian mountainous temperate forests in North-West part of "Stara Planina" mountain massif, bounded to the national border with Serbia on South.

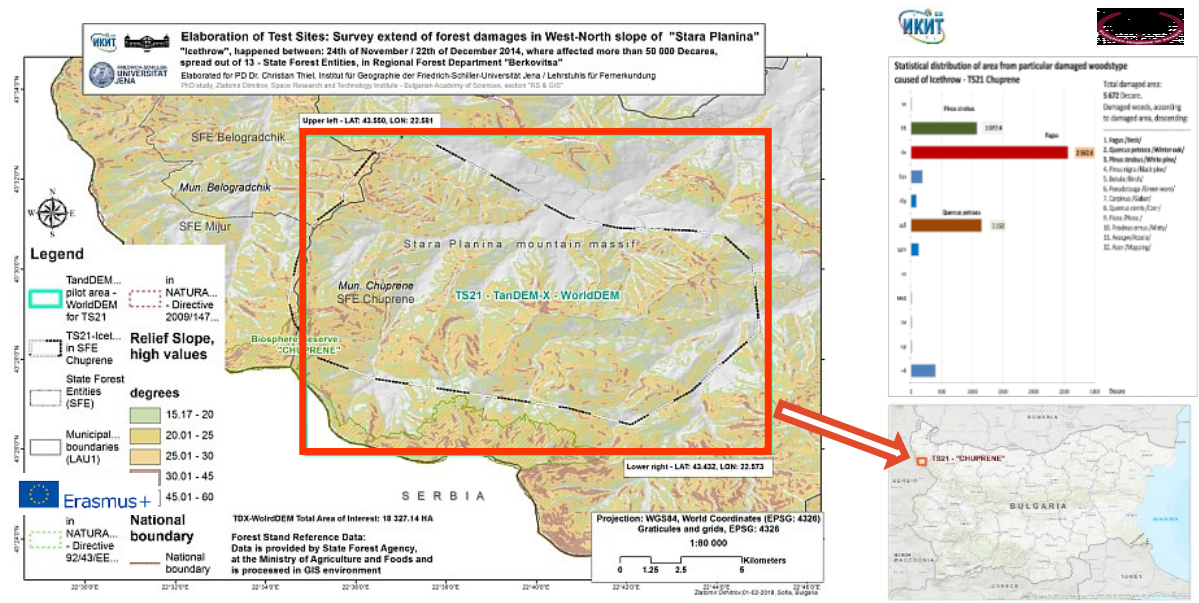

Fig. 3. The TS21 at SFE “Chuprene”; disturbed forest species with representative statistic. 
The test site is specifically selected to offer various environmental conditions, which comprise hilly and rugged terrain with strong topography and forest diversity - in the aspect of density and type. Also, the test site includes disturbed forest area with different percentages of damages on forest (from 20 up to $100 \%$ ), caused by severe Icethrow natural disaster happened at the end of 2014 . Deciduous forests are the main forest type, where main species diversity is presented by Winter Oak, Beck, Carpinus and other, showed on figure-3 (see, statistics chart).

\section{Meteo-situation:}

The meteorological condition at the time of acquisition is showing shows clear calm weather, with low winds; previous precipitation, relative humidity ab. $80 \%$ representing the mean value on vertical stratification; mean temperatures in the early morning was ab. $15{ }^{\circ} \mathrm{C}$. That highlights almost perfect weather conditions.

\section{Methods}

Package based software GAMMA $\odot$ is collection of programs for interferometric and differential-interferometric processing, with a high scale of output products, such as DEM or displacement maps $[15,16]$. In that study, following GAMMA modules are concerned - the Interferometry, Differential Interferometry and Geocoding (ISP / DIFF \& GEO) modules, Land Application Tools (LAT) module and Display (DISP) module. Modularity architecture of the software allows building of complex processing workflows, and accurate tracking of processing steps and debugging. The GAMMA release from 2018 was available at the Uni-Jena's cluster; release supports bistatic InSAR processing needed for TanDEM-X bistatic, and programme tools for proper import of digital elevation models (DEM) in GAMMA format. In particular, for accessing GAMMA executables, it was considered to develop two implementation lines with different method approaches. Both implementation lines use the same GAMMA executable tools. Therefore, the calculation of the components from the interferometric phase via both approach results in the same output. In spite, the methodology intends to test calculation of the phase components separately, along both implementation lines.

The first approach comprises the implementation of the GAMMA executables in Bourne Shell Linux environment (BASH), as a set of shell scripts. Primal aim here is to calculate the Topographic phase component derived from interferometric measurement. Considered benefits from using this approach are related to: flexibility related to environmental variables in shell-scripts, with the direct output in terminal from processing GAMMA executables, useful in error tracking. 
The second approach comprises the implementation of GAMMA functionality, using GAMMA - API in PyroSAR, which is a Python-based framework, developed by MSc John Truckenbrodt, at Friedrich-SchillerInoversität-JENA, Lehrstuhl für Fernerkundung. It is open source software framework for large-scale SAR based satellite data processing, with extensive functional capabilities [17]. The primary aim pursued in that approach is calculation of the Differential phase component, which allows the differential height between referent DEM and SAR measurement to be derived. Considered benefits from using this approach via PyroSAR GAMMA-API are related to: fast implementation in terms of object-oriented Python environment and fast access to image specifications in metadata. The idea of the both line approaches is illustrated in Fig. 4A, down below.

\section{Processing with GAMMA}

Whole processing flow is conducted via two implementation lines, as mentioned above, to test different approaches in accessing GAMMA package to process SAR data, illustrated in Fig. 4A. Both implementation lines include method approaches via BASH shell scripts, and via Python scripts using PyroSAR GAMMA-API. A wide range of scripts has been developed by the author in Linux/Shell environment and Python 2.7, according to the scope of the two implementation lines. Coding aims to automate the processing chain with GAMMA binaries. The processing flow begins with data import of the TanDEM-X bistatic SAR data in GAMMA format. Afterwards, both implementations include initial and advanced data processing using InSAR-advanced techniques. Output products from both lines are scaled into $4 \mathrm{~m}$ and $12 \mathrm{~m}$ pixel spacing.

\section{Data import in GAMMA}

On the first instance - first level processing, the raw TanDEM-X CoSSC bistatic data are imported into GAMMA file format; it consists of two files, where:

- The binary file, which contains the COSAR - 32-bit floating point BigEndian complex image data (FCOMPLEX), organised in row order starting from the first to the last one;

- ASCII parametric files (PAR) providing general CoSSC product annotation, such as sensor type, date, range samples and azimuth lines, range and azimuth pixel spacing in metres, incidence angle image center (ellipsoid), and Orbit State Vectors (OSV), and other.

Data import is performed using PyroSAR, GAMMA-API. On the first instance function - finder() is used to retrieve desired TDX - datasets. Afterwards, datasets are identified and imported as PyroSAR objects, where function - 
convert2gamma() is used to import the datasets in GAMMA format; scheme is drawn on Fig. 4B.

Afterwards, the reference DEM is imported in GAMMA. Non-additional correction is preliminary needed for the TanDEM-X DEM12, in terms of vertical datum (WGS84) or axis offsets (which is not the case with SRTM for instance); thus, the input GeoTIFF is directly imported in GAMMA. The delivered output is a 32-bit floating point binary file, and annotation constituted in a PAR-file giving information for the - lines/rows, datum, offsets, data format (e.g. REAL), and posting a resolution on latitude/longitude; the DEM-projection is according to the assumption in GAMMA - EQA coordinates [18].

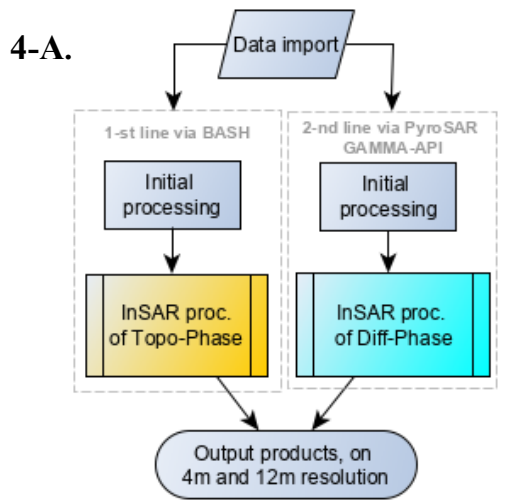

4-B.

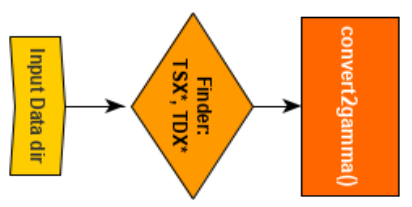

Fig. 4-A: (left) Basic scheme of the two-lines implementation, first via BASH scripting, and second via PyroSAR GAMMA-API in Python. 4-B: (right) Import of TanDEM-X datasets in GAMMA format, with PyroSAR/PyroSAR GAMMA API.

\section{Initial processing}

Initial processing workflow is executed prior to interferometric processing of SAR data. It aims pre-processing of imported bistatic SLC's for transmit/receive, in particular, as follows:

Calculate Offset - In the proposed processing chain offsets are calculated in purpose of the accuracy testing of the co-registration. This step generates a polynomial of offsets on range $(\mathrm{Rg})$ and azimuth $(\mathrm{Az})$, showed extreme accuracy with error of less than $1 / 10^{\text {th }}$ of a pixel.

Resample Slave on Master geometry - Considering the bistatic acquisitions this resampling could be omitted. Besides, resampling is performed as assurance against possible inaccuracies (e.g. presence of rugged terrain). 
Calculate multi-look factors, with respect to target resolution - Multi-look factors are calculated in a separately coded function, according to target-resolution, incidence angle, and $\mathrm{Rg} / \mathrm{Az}$ pixel spacing in particular TanDEM-X imagery. Varieties of target-resolution values with ML-factors are tested to produce balanced solution of pixel spacing on $\mathrm{Rg}$ and $\mathrm{Az}$. In target-resolution of $4 \mathrm{~m}$ corresponding to ML-factors of 2, resulted in ground range pixel spacing on $\mathrm{Rg}=$ $3.864 \mathrm{~m}$, and on $\mathrm{Az}=4.794 \mathrm{~m}$, where $\mathrm{Rg}-\mathrm{Az}-$ delta $=-0.930 \mathrm{~m}$. This is close to the critical delta difference, where higher values were found to introduce problematic geocoding. A good solution is derived aiming target-resolution of $12 \mathrm{~m}$, where derived ML-factors are giving:

$\mathrm{Rg}=11.988 \mathrm{~m}$, and on $\mathrm{Az}=11.581 \mathrm{~m}$, with the delta $=0.406 \mathrm{~m}$.

Multi-Looking - Higher multi-looking is better to reduce multiplicative speckle noise which bias the InSAR measurements, with respect to resolution. Multi-looking is performed according to the calculated ML-factors and the targetresolution. The produced outputs are the Multi-Looked Intensities (MLI).

Calculate Over-Sampling Factors for LUT - Over-sampling factors (OSF) are used as scaling factors for the DEM-pixels, in the sake of resampling them to a size of resolution cell in SAR image. The postings on LAT/LON provided in the DEM-PAR file are used.

Calculation of LUT, refinement (optional) - The generation of Look-up table is essential for geocoding process, and it is generated with the provided DEM and OSV of SAR (in PAR file), along the calculated OSF. The necessary refinement of the LUT as an optional step is done in the first-line implementation via BASH.

Resample DEM to Range-Doppler Coordinates (RDC) - In this step, the transformation of the corresponding AOI from the DEM, from map to radar geometry (RDC) is performed, using LUT.

Radiometric calibration - Both MLI are radiometric calibrated to sigmanought $\left(\sigma^{0}\right)$, which express radar-cross section for each pixel, physically measured in $\mathrm{dB}$, derived from the geometry of the acquisition held in OSV in PAR file. In purpose of normalisation, the ellipsoid-based pixel reference area in ground range is outputted.

Normalization and Terrain Flattening - The final step comprises the normalisation of radar cross-section $\left(\gamma^{0}\right)$, and compensation of the local topography based on method by David Small [11]. After the calculation of pixel-scattering area - representing the backscattered energy from the ground with respect to local topography, the actual Normalisation and Terrain-flattening are calculated, whilst perform eliminating the effect of topography. Terrain-flattening enhance the analysis based on intensities. For this purpose, the layover, shadow and incidence angle maps are used. Please, refer to Figures-5 and 6, down below. 
Implementation via BASH - The first line implementation of the initial workflow is performed via BASH scripts, including whole steps above. The direct scripting approach here allowed LUT-refinement - to overcome spatial misalignment, because of problems found after geocoding. An important step in BASH scripting during Multi-Look was storing the sizes of the MLI images as BASH variables, needed further in processing. The workflow is presented on following Fig. 5:

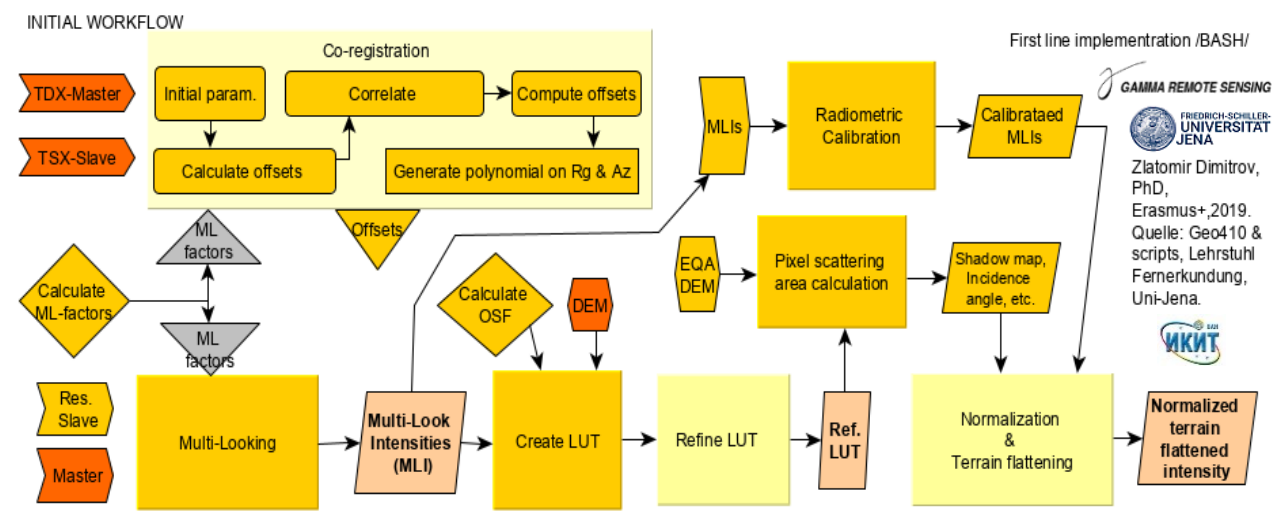

Fig. 5. Initial workflow at first line implementation constituted as Linux BASH scripts, which control directly GAMMA software binaries. Content - reddish inputs: TDX products; - yellow inputs: resampled slave over master / MLI or CMLI / DEM in SAR geometry; - yellow diamond: functions; - yellow triangles: intermediate parameters; - greyish triangles: input BASH variables; yellowish rectangles: processing steps; light yellow - complex processing steps; rose objects: outputs from processing in 4 or $12 \mathrm{~m}$ resolution scale.

\section{Implementation via PyroSAR GAMMA-API:}

The second line implementation of the initial workflow is performed via Python scripts accessing GAMMA-API in PyroSAR and additional functions available in the framework.

It differs from the first line implementation, where LUT refinement is excluded, due to unavailability within GAMMA-API. Workflow is compact and performed faster, than the first one; object-oriented Python environment allowed easy transition of the input/output function parameters and access to GAMMAAPI. Easy access to SAR imagery metadata was provided by ISPar class in PyroSAR. Also, coding of workflow was facilitated by the build-in functions in Python. Nonetheless, the general negative issue was found, resulting in the misalignment of the geocoded output products, due to possible lack of LUT refinement. Please, refer to Fig. 6. 


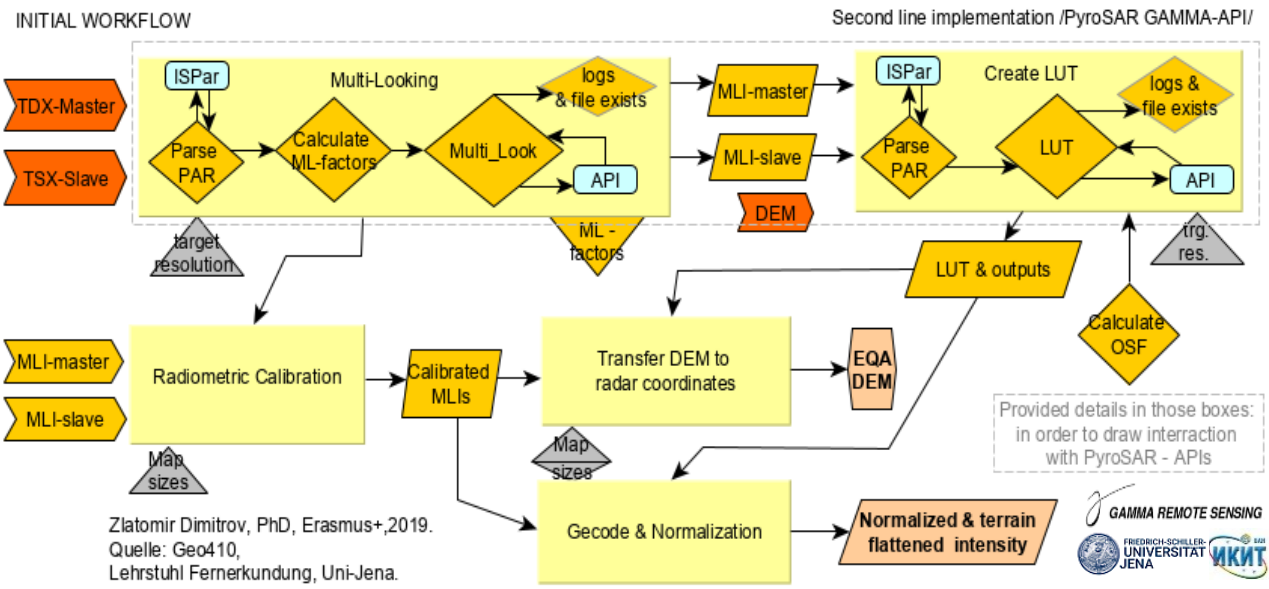

Fig. 6. Initial processing at the second implementation line constituted as Python scripts using PyroSAR GAMMA-API, which creates access to GAMMA executable binaries. Content: - bluish rectangles: GAMMA API/PyroSAR functions; - yellowish diamonds: Module functions; - light yellowish rectangles: processing steps *in dashed contour - details provided; - yellowish trapezoid: function output as a Python object; - rose objects: outputs.

\section{InSAR Processing}

Interferometric processing of the CoSSC bistatic pair derives the Interferometric phase components - topographic and differential phases, where height products along radar beam to be calculated. Approaches in GAMMA for baseline, coherence and phase-to-height calculations are similar, but rather different in the scope of interferometric calculations. On the other side, interferometric workflows in both implementation lines also differ each other. Therefore, workflows are presented separately according to both implementation lines.

\section{InSAR processing of Topographic phase - implementation via BASH:}

For the Topographic phase InSAR processing several BASH scripts were developed, constituted of:

Raw-Interferogram calculation - In this step, interfering with the bistatic pair (CoSSC) is performed using Master and resampled Slave, using OSV, offsets, and multi-look factors on $\mathrm{Rg}$ and $\mathrm{Az}$. The raw Interferogram is strongly modulated by the range-dependent fringes - Flat-earth phase component from the interferometric phase. Results are displayed from GAMMA using MLI overlay. 
Baseline estimation - For Baseline calculation two methods are used - by using OSV (less accurate), and based on the fringe-frequency method (FFT) from the raw Interferogram (more accurate). Both are compared.

Interferogram flattening - Removal of the Flat-earth phase component is performed, using OSV and baseline, where the range-dependent fringes are removed. Results analysis showed a strong modulation, apparent over flat areas.

Coherence estimation - The interferometric correlation is systematically high, due to the bistatic scenario acquisition, whereas main decorrelation in behind is presented by the volumetric decorrelation. This is so, due to the baseline decorrelation, resulting from the different location of the active phase centers, within the resolution cell. Coherence is calculated using adaptive approach and fixed window of max 9 pixels.

Adaptive filtering - Adaptive filtering of the complex Interferogram is performed, in order to filter out the presence of speckle noise. Results analysis showed well performance, with reduced phase noise also.

Phase unwrapping - Phase unwrapping is performed on three steps, solving ambiguities of discrete distribution of the phase, ranging within the interval $[0,2 \pi]$. Firstly, areas with low coherence are masked out - those are mountainous terrain AND areas with a high value of biomass. As observed over the test site, due to geometric distortions, such areas are very common. Secondly, the minimumcost-flow approach is applied over the filtered complex Interferogram, using triangular-mesh method in GAMMA, by provided validity mask. Finally, the 2Dfiltering is executed to fill the rest small gaps.

Phase-to-Height - To estimate the height from the unwrapped Interferometric phase, two essential steps are performed in advance: Improving the baseline using ground control points, and inversion of the phase-to-height. Due to high scale reference DEM precise GCPs in terms of location and height are available, allowing the extraction of those GCPs from the reference TanDEM-X DEM. Afterwards, improving the Baseline is performed firstly by providing the unwrapped interferogram, and secondly, by providing GCPs and Baseline. The conversion of the unwrapped phase-to-height is finally performed considering geometry along radar beam, which outputs the height map in metres.

Geocoding \& export to GeoTIFF - Final steps from InSAR workflow are geocoding and exporting results to GeoTIFFs. Three general outputs are considered to be finally geocoded and exported - Multi-Looked-Intensities (MLI) of master TDX image, Coherence, and Height map from the topographic phase - high resolution DEM.

Important moment in geocoding concerns LUT accuracy used to transfer as accurately as possible the Range-Doppler Coordinates to Map-geometry. In proposed processing the geodetic world coordinate system (EPSG:4326) with 
vertical datum WGS 84 is used, stored in 24-bit GeoTIFF files. Whole datasets are displayed and overlaid in GIS software examined for consistency after InSAR processing. Please, refer to Fig. 7, as follows.

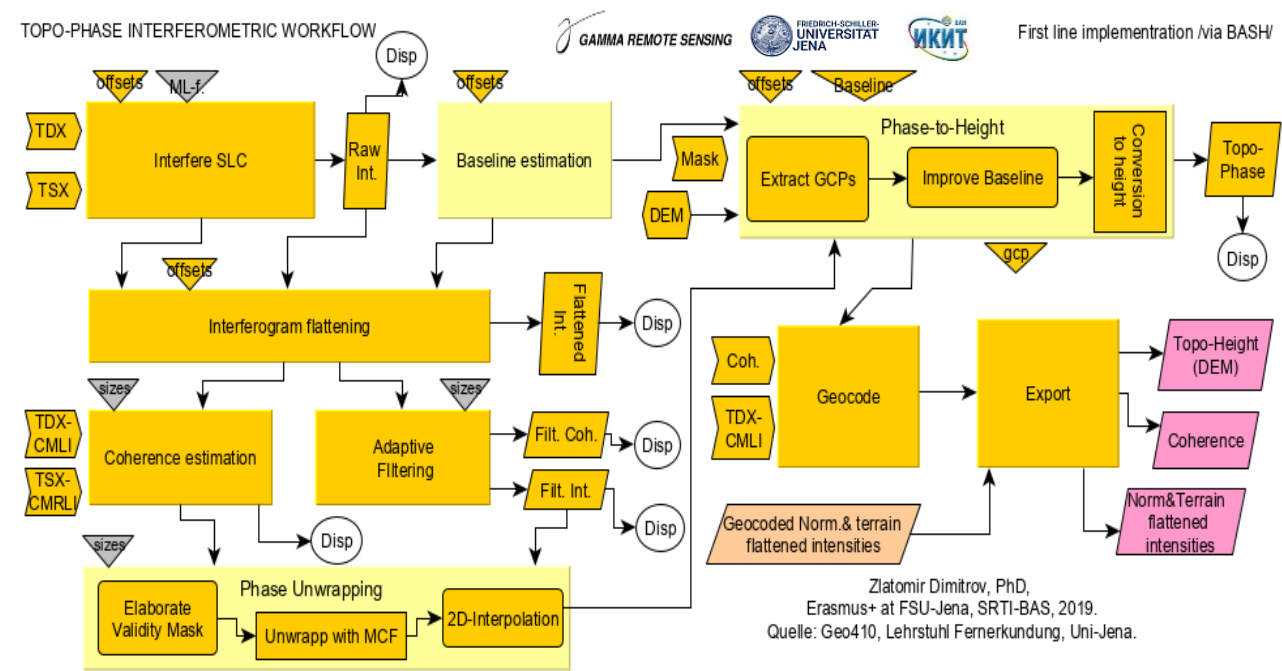

Fig. 7. InSAR-workflow at first line implementation constituted as Linux BASH scripts. Contentreddish/yellow inputs: TDX products/Calibrated MLI / DEM in SAR geometry, Low coherence mask, Coherence, Filtered coherence, Filtered Interferogram etc.; - yellow triangles: intermediate parameters; - greyish triangles: input BASH variables, MLI-width/height; yellowish rectangles: processing steps; light yellow - complex processing steps; magenta objects: final outputs from InSAR processing in 4 or $12 \mathrm{~m}$ resolution scale in GeoTIFF. White circles represent intermediate display via DISP-GAMMA module, from X-server in Linux environment.

\section{GAMMA-API}

InSAR Processing of Differential Phase - implementation via PyroSAR

The purpose for calculation of Differential phase originally derives differential height, which could be linked directly with the canopy height, in case of the bistatic data acquisitions from TanDEM-X [19]. The main idea of this approach considers existence of LiDAR DTM, from which the ground phase to be simulated and then subtracted from the interferometric phase. Thus, calculated differential height is directly related to the forest canopy height, determined by uncertainties of the acquisition!

Despite the differential phase calculation, the second objective in this implementation line considers the powerful GAMMA-API from PyroSAR framework to be tested and utilised for TanDEM-X interferometric processing. Therefore, a project in a Python environment was developed, including one main script with a module, where embedding the PyroSAR GAMMA-API functionalities. The, SAR imagery and supporting data are Python objects, thus 
allowing marvellous flexibility considering processing, manipulation and accessing metadata parameters. The InSAR processing in the second line implementation includes some of the processing steps already described in the above, but includes the following steps: method;

Baseline estimation - estimation differs here, based only on the FFT

Calculate InSAR parameters - A special function in Python was developed, where to calculate the specific parameters relevant to the InSAR pair ambiguity height $(H O A[\mathrm{~m}])$ and vertical wave number $(k z[\mathrm{rad} / \mathrm{m}])$;

Calculate raw interferogram - Intentionally for reference purposes and analysis;

Simulate unwrapped InSAR phase - High-scale unwrapped phase simulated from the reference TanDEM-X DEM, to be used in the calculation of the differential interferogram from TanDEM-X bistatic acquisition;

Calculate differential interferogram - The unwrapped differential interferogram is directly calculated in GAMMA functionality via GAMMA-API, using simulated unwrapped phase. Thus, differential interferogram is representable to differences between the vertical position of the phase centers and height from the reference TanDEM-X DEM. It also includes atmospheric components and phase noise - a matter of further filtering.

Coherence estimation and adaptive filtering, Phase-to-Height, Geocoding \& export to GeoTIFF - Coherence estimation, adaptive filtering, conversion of phase to height along the radar beam and geocode and export is the same as stated in the first line, but here coding is facilitated by the ease of object orientated Python coding and benefits from the GAMMA-API. Results are successfully exported to Geo-Tiffs. Please, refer to Fig. 8, as follows. 


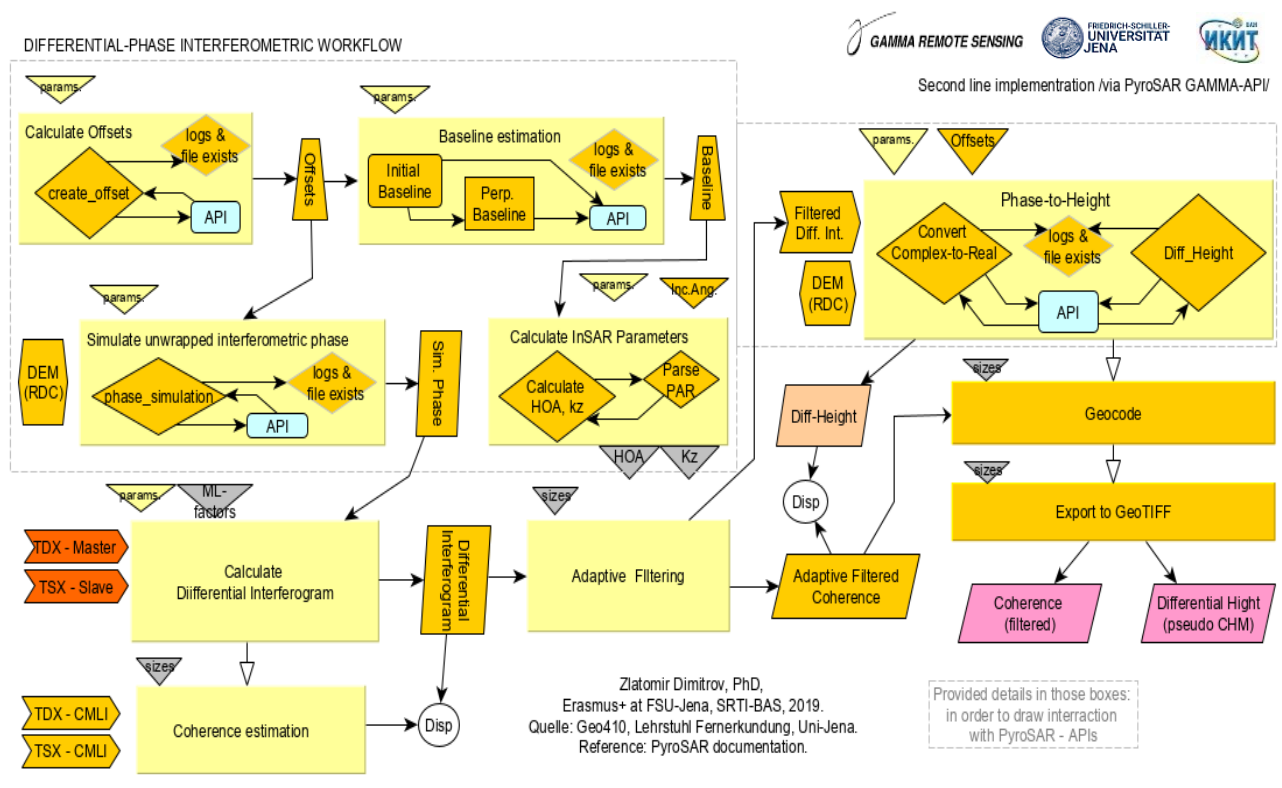

Fig. 8. Interferometric workflow of the Differential-Phase calculation with PyroSAR GAMMA-API, where: bright yellow boxes-processing steps *in dashed contour - details provided; yellow diamonds (borders) - functions of main script/module in Python environment; blue box reference to PyroSAR GAMMA-API / other functions; yellow diamonds (grey border) - set of Module script functions; grey rectangles - output/input variables from/to functions; red inputs - input TDX/TSX CoSSC in GAMMA; white ellipse - display functionality via GAMMA display tools within Python; - yellowish boxes: function output as a Python object; - Magenta rhomboid: outputs geocoded products in GeoTIFF.

\section{Results and analysis}

Calculated output products have been processed in two scale of spatial resolution $-4 \mathrm{~m}$ and $12 \mathrm{~m}$. The first spatial resolution scale is determined from the desire for high resolution InSAR products from TanDEM-X, whilst second one is especially considered to have an overlapping of the resolution cells, between MLI and the reference DEM from TanDEM-X. It is intentionally for differential phase analysis. During processing monitoring of intermediate results is performed in the first line implementation by providing graphical output in BASH via GAMMA, by the display tools, such as - dishgt, dis2hgt, dis2rmg, dis2mph, etc., for educational and developing purposes, and for adjusting the variety of additional input parameters.

Thus, the GAMMA output consists of the following dataset - Coherence, Filtered Coherence with ADF, Differential-Height (pseudo Canopy Height Model CHM) calculated from Differential phase, Differntial-phase, Topographic-Height 
(DEM), Normalized \& terrain-flattened Intensity and Averaged Intensity between Master and Slave.

\section{Analysis on output products with resolution scale of -4 [m]}

Coherence - Both interferometric coherence amplitudes are showing strong dependency from the topography, where areas with geometric distortions, coherence is low, nonetheless filtering is applied. Also, the relative dependency from volumetric decorrelation is also present, thus showing forest areas.

Topographic Height - Comparing the results from the calculated Topophase, shows conformity along the TanDEM-X DEM, where the observed differences could be mainly related to errors in phase unwrapping, and differences in heights of the phase centers. Here, must be aware, that the TanDEM-X DEM is exclusively advanced product with very high accuracy, comprising more than 38acquisitions in a point of Earth. This is a result of covering various seasonality and meteorological conditions over the canopy, different HOA, different orbit and look angles, thus so to cope with the complicity of processes of the backscatter from natural targets. It should be noted, that the reference height from the DEM from TanDEM-X data is closer to the canopy height, than the reference DEM.

Differential Height - The output product of Differential height held within the second implementation line via PyroSAR GAMMA-API, is not representative of CHM due to no ground phase is known, but rather some mean reference canopy height is used as the reference ground phase; thus, this product is much to support the analysis on the canopy, and to tell what is the difference between referent DEM and the height of the TanDEM-X scattering phase centers from particular acquisition. Moreover, phase unwrapping is obeyed, because of the proposed assumption in the literature [20], aiming to improve accuracy over mountainous areas. Values of the Differential height are showing inconsistency with the canopy height, rather, as already stated a measure of differences. Most negative values reside in areas where the interferometric phase is noisy and coherence is low - e.g., geometric distortions. Nonetheless, resulted product shows conformity with the type of canopy, where differential height is homogenous non-forest areas, and rather heterogeneous over forest areas.

Normalised and Terrain Flattened Intensity - Terrain flattened and normalised intensity fosters amplitude analysis in the rugged terrain. Topography influence is almost eliminated, were intensity values stayed almost invariant to the slope orientation along the radar beam. As expected, a drop in backscatter is observed over forest areas. Contrary, high backscatter is observed over some bare fields and of course over urban areas (e.g. houses in Chuprene).

Averaged de-Speckled Intensity - The averaged intensity with applied Frost-speckle filter with a window of $7 \times 7$ showed good elimination of speckle, and allowed consistent amplitude analysis. Radar shadows frustrate analysis, but 
represents the real influence of the mountainous terrain. The GAMMA output product set is presented as follows:

Table 2. Output products from GAMMA (both implementation lines), with a spatial resolution of $4 \mathrm{~m}$

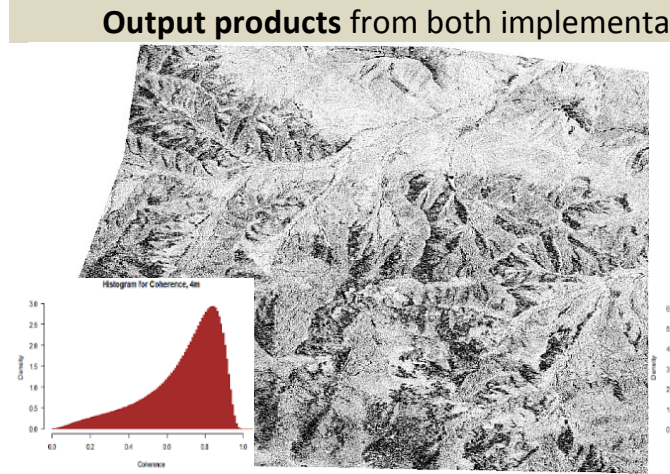

A) Coherence

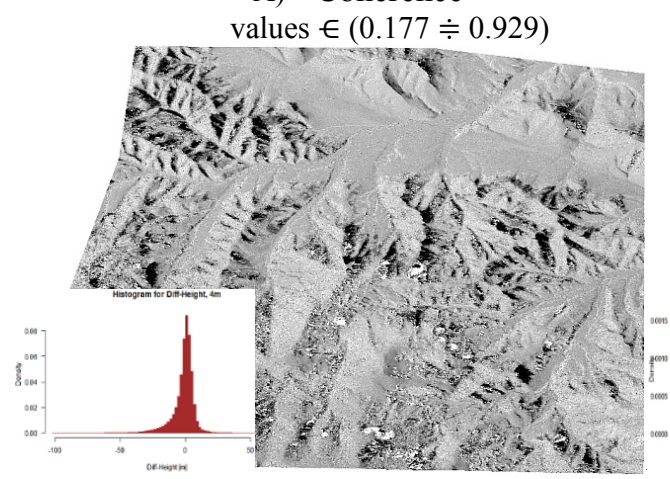

B) Differential-Height

values $\in(-27.125 \doteqdot 11.379)[\mathrm{m}]$

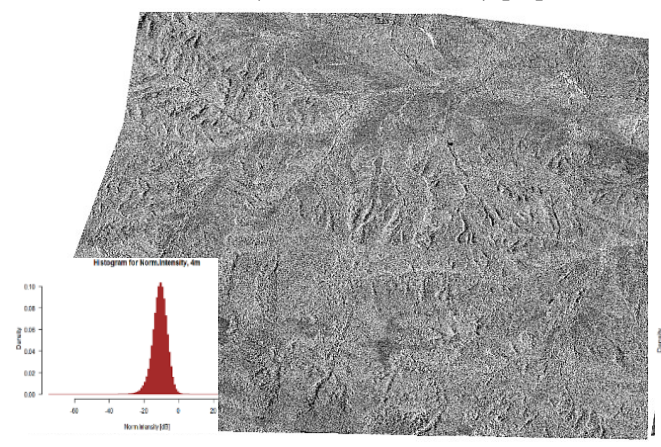

C) Normalized and Terrain flattened Intensity

values $\in(-20.816 \doteqdot-3.127)[\mathrm{dB}]$

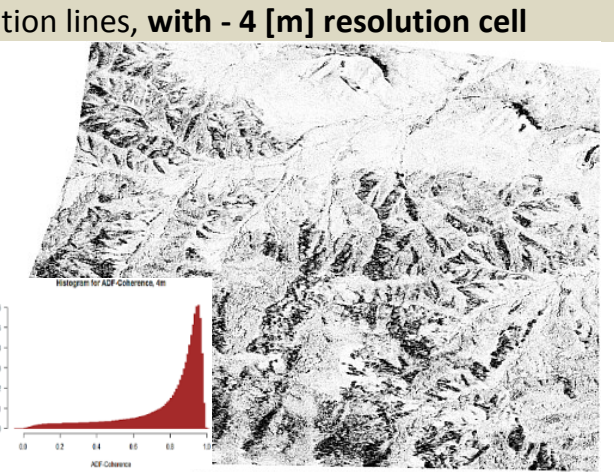

D) Filtered Coherence with ADF values $\in(0.134 \doteqdot 0.976)$

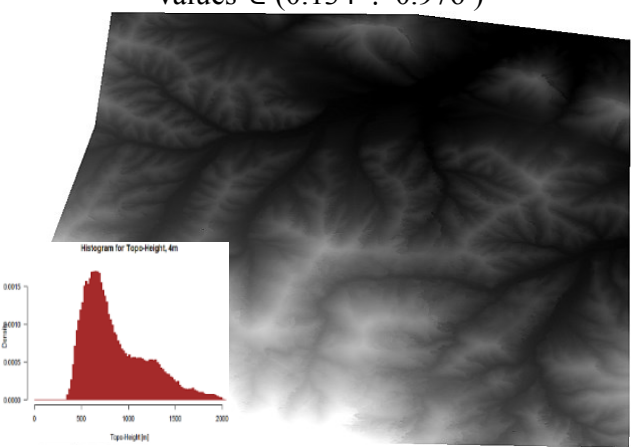

E) Topographic-Height values $\in(421.958 \doteqdot 1726.19)[\mathrm{m}]$

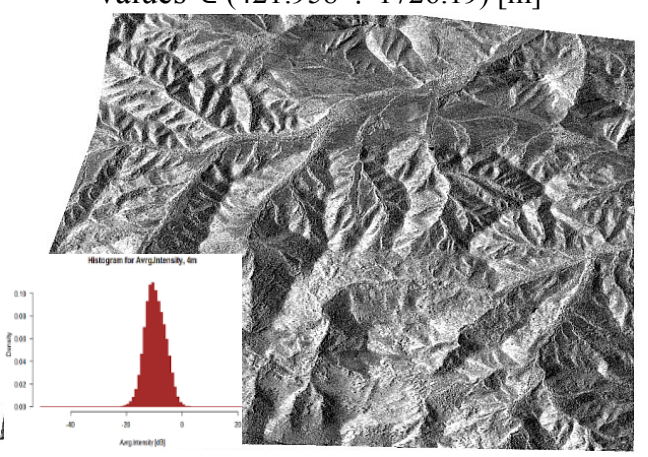

F) Averaged de-Speckled Intensity values $\in(-16.601 \doteqdot-2.438)[\mathrm{dB}]$ 


\section{Analysis on products with resolution scale -12 [m]}

The output products are showing almost the same dependencies, as stated above, with small differences with respect to spatial resolution and phase unwrapping for differential height. Coherence amplitude shows better relation to the volumetric decorrelation over canopy. Geometric distortions are almost masked out with homogenous low values. Agricultural areas are well distinguished, also man-made objects. Analysis of the Differential height shows relative improvement with respect to the $4 \mathrm{~m}$ - product. Sensitivity to forest areas is better, with a smaller range of values showing better consistency with the assumption this to be measure for differences between reference height and height of the phase centers, which could be related to problems in forest areas - such as forest disturbances. In spite, at the $12 \mathrm{~m}$ - products discrete values showing phase jumps are observed, because phase unwrapping is omitted.

Table 3. Output products from GAMMA (second implementation line), with a spatial resolution of $12[\mathrm{~m}]$

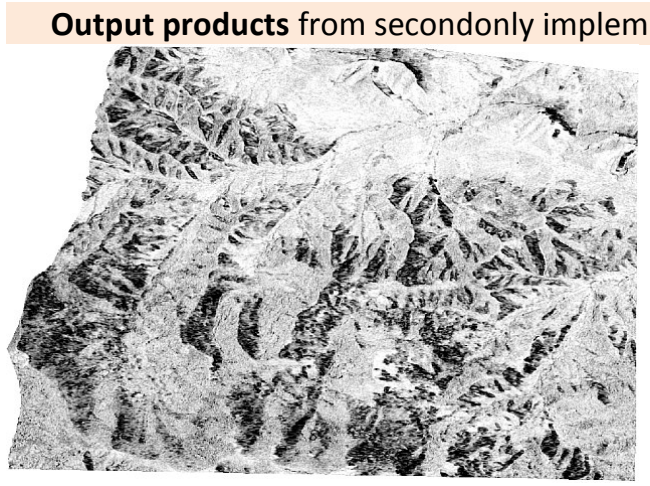

G) Coherence

values $\in(0.158 \doteqdot 0.893)$

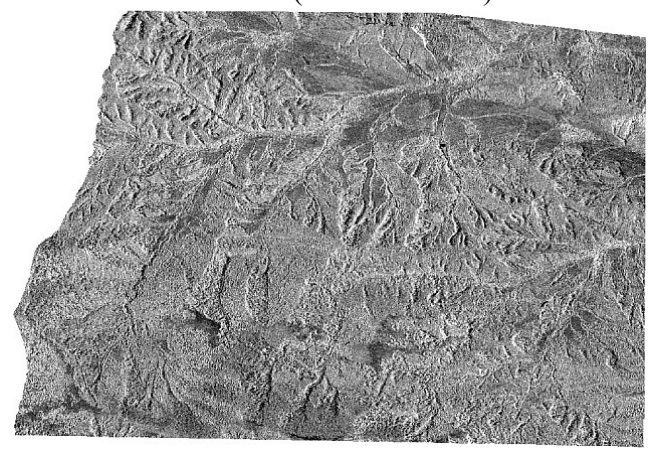

H) Normalized and Terrain flattened Intensity values $\in(-16.476 \doteqdot--4.449)[\mathrm{dB}]$

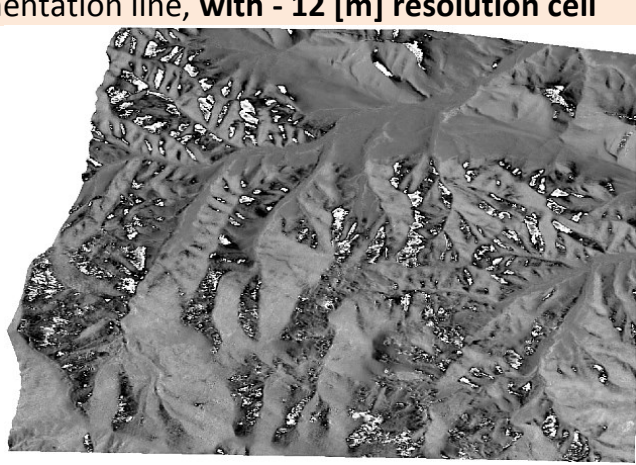

I) Differential-Height values $\in(-19.113 \doteqdot 12.672)[\mathrm{m}]$

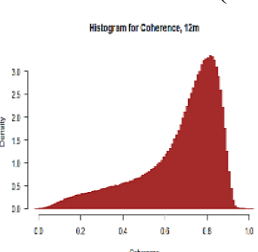

Hist. of Coherence,12m

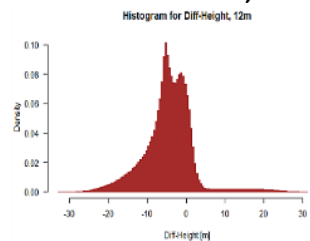

Hist.of Diff.Height,12m

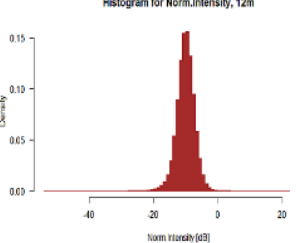

Hist. of Intensity,12m 


\section{Discussion}

Distinctly, most advantage of GAMMA software package is that it offers various functionalities giving possibility to produce different components of the Interferometric phase. Nonetheless, small discussion is useful to be made considering frustration and difficulties on both implementation approaches. Main difficulties at first approach (first implementation line), are related to the enormous parameters that should be passed to the GAMMA executables. This expects punctuality and precision during coding; in spite, freedom to any functional needs is available. Considering second approach (second implementation line), difficulties are much reduced, with respect to the benefits from object-oriented nature of the PyroSAR framework, that resides in Python environment. The only minus here is that customisation of functionality needs coding, which puts inspiration to further contribute to this useful framework, where to expand its functionality.

During the utilisation of the TanDEM-X bistatic data over mountainous forest areas, analysis in GIS environment of the output interferometric products showed particular inaccuracies in respect of terrain slope and the type of land cover. There are two main reasons, why products derived from topo- and diffphases from this processing flow were not completely accurate. First - considering topographic phase, data acquisitions in X-band are preliminary backscattered from smaller objects comparable to the wavelength, thus resulting in backscatter from the top of the forest canopy. This obliges filtering after phase unwrapping, because of increased error probability density. Second - considering the differential phase, concerns the available reference TanDEM-X DEM12, which is influenced by the canopy height due to the property of short microwaves (e.g. X-band), which saturates in the higher biomass, thus extinction is higher and therefore it does not represent the ground phase. Moreover, the bistatic acquisitions are showing big sensitivity to geometric distortions in rugged terrain, thus those areas are ambitious to be interpreted.

It is considered further development of this study in case of available LiDAR reference acquisition providing the ground phase, or the topography of the forest floor (e.g. DTM). Thus, the differential phase method approach (second implementation line) could derive the realistic canopy height based on differential phase, which is truthfully utilization of differential SAR interferometry based on $\mathrm{X}$-band SAR data from bistatic acquisition.

\section{Conclusion}

This study demonstrated utilization of interferometric processing of TanDEM-X bistatic SAR data acquisitions with software GAMMAC. It is based on two different coding approaches, resulting in interferometric outputs on two different resolution scales of 4 and $12 \mathrm{~m}$. 
First implementation line via BASH scripts allowed better track of intermediate results and detailed scripting capabilities. Whilst, utilisation of TanDEM-X bistatic processing via PyroSAR, GAMMA-API showed very good feasibility and fast scripting, via the object-based environment in Python. Also, the powerful PyroSAR framework with the GAMMA-API allowed ease of coding into very common Python environment, allowing object-based approach in processing of SAR data. A comparison of both implementation lines (e.g. via BASH and via PyroSAR GAMMA-API) showed that they complement to each other, in terms of the expected result and processing chain.

Analysis of interferometric outputs on both resolution scales showed various performances. The interferometric processing of topographic phase showed high fidelity in a $4 \mathrm{~m}$ resolution scale, and utilised well the bistatic acquisitions from TanDEM-X in rugged terrain, with the presence of various forest canopy. Multi-looking of up to $12 \mathrm{~m}$ of the high-resolution TanDEM-X bistatic data revealed better sustainability of the interferometric products, in mountainous forest areas with strong topography. Considering differences in reference height of the high scale TanDEM-X DEM12 and the vertical position of the active phase centers from the TanDEM-X acquisition, omitting phase unwrapping gave better results, than high-resolution products of $4[\mathrm{~m}]$ with phase-unwrapping applied.

Finally, could be concluded that utilisation showed that the GAMMA package offers extreme flexibility in solving SAR remote sensing tasks offering professional approach in applying interferometry on complex land cover, especially over mountainous forest areas with the presence of topography. It is least likely such performance to be achieved via other available graphical based software, based on author's experience.

\section{Acknowledgement}

The TanDEM-X CoSSC data was provided from DLR in the frame of CoSSC data proposal, where the TanDEM-X DEM12C high resolution DEM product in the frame of a DEM proposal.

Elaborated scripts by the author follow up the scope of the course GEO410 'Programmierung in der Fernerkundung (GAMMA)', held in the frame of Erasmus + exchange at Friedrich-Schiller-Universität - JENA, Lehrstuhl für Fernerkundung.

During development, I wish sincerely to thank John Truckenbrodt, for the precious help over PyroSAR, installation and usage of GAMMA-API, and the help of the Python debris. I wish to thank to Dr. Christian Berger from the Lehrstuhl, for providing me with their available base scripts that facilitated the development of the BASH scripts.

Also, mine sincere thanks to the Bulgarian Academy of Sciences, and ERASMUS+ programme that gave the chance to visit FSU - JENA, Lehrstuhl für Fernerkundung, and to Dr. Christian Thiel who invited me, prof. Christiane 
Schmullius the head of the Lehrstuhl; the TanDEM-X Science Team from DLR the Microwave and Radar Institute for providing me with this extraordinary TanDEM-X data. Finally to my tutor prof. Eugenia Roumenina, and my father prof. Dobri Dimitrov, and to all scientists I have met in Jena, who showed a friendly support and where always ready to answer my questions.

\section{References}

1. Bamler and Hartl. Synthetic Aperture Radar Interferometry. Inverse Problems, 14. 1998.

2. Richards, M. A. Beginner's Guide to Interferometric SAR Concepts and Signal Processing. IEEE A\&E Systems Magazine, 2007, 22, 9.

3. Hajnsek, I., et al. Tropical-Forest-Parameter Estimation by Means of Pol-InSAR: The INDREX-II Campaign. IEEE, 2009, 47, 2.

4. Dimitrov, Z. Potential of POLinSAR in survey of forest ecosystems. Conference paper, In: Proceedings of $12^{\text {th }}$ Scientific Conference with International Participation-SES2016, Nov-2016, URL: http://space.bas.bg/SES/archive/SES\%202016 DOKLADI/3 Remote\%20Sensing/15 Dimitrov.pdf. (In Bulgarian)

5. Thiel, C. and Schmullius, C. The potential of ALOS PALSAR backscatter and InSAR coherence for forest growing stock volume estimation in Central Siberia. FSU-Jena, Elsevier, RSE-173, 2016.

6. Wilhem, S., et al. Large Area Mapping of Boreal Growing Stock Volume on an Annual and Multi-Temporal Level Using PALSAR L-Band Backscatter Mosaics. MDPI, 2014, 5.

7. Santoro, M., et al. Forest growing stock volume of the northern hemisphere: Spatially explicit estimates for 2010 derived from Envisat ASAR. Elsevier, RSE-168, 2015.

8. Martone, M., et al. Volume Decorrelation Effects in TanDEM-X Interferometric SAR Data. IEEE, 2016, 13, 12.

9. Caicoya, A. T., et al. Large-Scale Biomass Classification in Boreal Forests With TanDEM-X Data. IEEE, 2016, 54, 10.

10. Kugler, F., K. Papathanassiou, and I. Hajnsek. Pol-InSAR Techniques Forest Characterisation with TanDEM-X. HR Institute at DLR, POLinSAR-2013 ESA, Frascatti, 29 January 2013.

11. Small, D. Flattening Gamma: Radiometric Terrain Correction for SAR Imagery. IEEE, 2011, 49, 8 .

12. Krieger, G., A. Moreira, H. Fiedler, I. Hajnsek et al. TanDEM-X: A Satellite Formation for High-Resolution SAR Interferometry. IEEE, 2007, 45, 11.

13. Shulze, D., M. Zink, G. Krieger, J. Böer, and A. Moreira. TanDEM-X Mission Concept and Status. DLR, 2008.

14. Krieger, G., et al. TanDEM-X - A Satellite Formation for High Resolution SAR Interferometry. DLR, Microwave and Radar Institute, 2005.

15. GAMMA Software Information, v1.4, 22-Feb-2019, GAMMA Remote Sensing Research and Consulting AG, 2019.

16. GAMMA SAR AND INTERFEROMETRY SOFTWARE, v2.1, TS, UW, CW, GAMMA Remote Sensing Research and Consulting AG, Sep. 2006.

17. Truckenbrodt, J., F. Cremer, I. Baris, J. Eberle. PyroSAR - A Framework for Large-Scale SAR Satellite Data Processing. FSU-Jena, Conference paper, DOI: 10.13140/ RG.2.2.16424.83206, May 2019. 
18. Santoro, Maurizio, (Cartus, Oliver). Course GEO 410, Uni-JENA, SS 2019. "Lection-1. Geocoding with the DIFF\&GEO module“. Slide 10/12. Gamma Remote Sensing AG, 17.Nov.2017.

19. Santoro, M., (Cartus, Oliver). Course GEO 410, Uni-JENA, SS 2019. "Lection-5. Interferometric processing of TanDEM-X with ISP/DIFF\&GEO/LAT“. Slides 37-39. Gamma Remote Sensing AG, 17.Nov.2017.

20. Santoro, M., (Cartus, Oliver). Course GEO 410, Uni-JENA, SS 2019. „Lection-2. Interferometric processing of ALOS PALSAR for generating a Digital Elevation Model“. Slides 37-39. Gamma Remote Sensing AG, 17.Nov.2017.

\section{ИНТЕРФЕРОМЕТРИЧНА ОБРАБОТКА ЧРЕЗ СОФТУЕРА"GАММА” НА РАДИОЛОКАЦИОННИ ИЗОБРАЖЕНИЯ ОТ SAR, OT HЕМСКАТА СПЪТНИКОВА БИСТАТИЧНА СИСТЕМА - ТАNDЕМ-Х}

\section{3. Димитров}

\section{Резюме}

Интерферометричният анализ (InSAR) на радиолокационни изображения от SAR c цел пресмятане на различните компоненти на интерферометрич-ната фаза е сложна задача в дистанционните изследвания. Софтуерьт GAMMA предлага най-добрата гъвкавост и професионален подход. Тази статия пред-ставя пилотно приложение на софтуера GAMMA посредством две работни вериги, за пресмятане на топографската и диференциалната фази от радарни изображения с бистатична конфигурация на заснемане, в микровълнов канал - "X" от немската спътникова система TanDEM-X, на немската космическа агенция - DLR. Използван е тестови район в северозападния дял на Стара Планина. Изследвани са два подхода на обработка - чрез „ВАSH“- скриптове с директен достьп до команди от GAMMA; - чрез многофункционалния пакет „PyroSAR“ и съответния му приложен програмен интерфейс - GAMMA-API. Изходните продукти са представени с $4 \mathrm{~m}$ и $12 \mathrm{~m}$ пространствена разделителна способност (ПРС), като показват различни предимства при условия на релеф и висока надземна биомаса. Едно от заключенията на изследването е, че из-ползването на GAMMA дава най-добрата гъвкавост и точност в изчисляването на различни компоненти на интерферометричната фаза, особено в планински територии. 